Artículo de investigación

\title{
EL POLEN APÍCOLA COMO PRODUCTO FORESTAL NO MADERABLE EN LA CORDILLERA ORIENTAL DE COLOMBIA
}

\author{
Bee pollen as non-wood forest product in the eastern Andean highlands of \\ Colombia
}

Palabras clave: apicultura, bosques de roble, melisopalinología, Quercus humboldtii.

Key words: beekeeping, oak forests, melissopalynology, Quercus humboldtii.

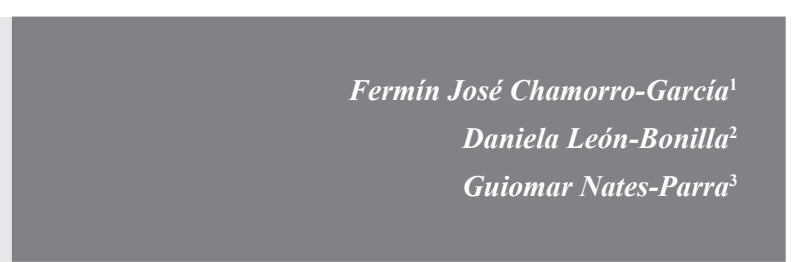

llevar a cabo prácticas de conservación del bosque dentro del manejo apícola.

\begin{abstract}
The Andean forests of the Eastern Andean highlands of Colombia have a high conservation priority given the vulnerable condition of species such as Quercus humboldtii (Fagaceae) that inhabit these ecosystems. Beekeeping is regarded as an alternative activity that could play a role in the conservation of Andean forests, but little is known about how the floras of these ecosystems contribute to honey and bee pollen production. We analyzed the contribution of Andean forests to bee pollen production, given the productive potential and commercial importance of this product. Pollen analyses were performed on 25 samples from apiaries near Andean forests located in the states of Cundinamarca, Boyacá and Santander. We found that $Q$. humboldtii is an important source of pollen with high potential for monofloral bee pollen production. In addition, bees collect pollen from other Andean forests species such as Weinmannia tomentosa, Viburnum spp. and Morella spp. Utilization of bee pollen could lead to incentives
\end{abstract}

Laboratorio de Investigaciones en Abejas (LABUN), Departamento de Biología, Universidad Nacional de Colombia, Sede Bogotá. Bogotá, Colombia. fjchamorrog@unal.edu.co. Autor para correspondencia.

Laboratorio de Investigaciones en Abejas (LABUN), Departamento de Biología, Universidad Nacional de Colombia, Sede Bogotá. Bogotá, Colombia.daleonb@unal.edu.co

3 Laboratorio de Investigaciones en Abejas (LABUN), Departamento de Biología, Universidad Nacional de Colombia, Sede Bogotá. Bogotá, Colombia.mgnatesp@unal.edu.co 
to carry out forest conservation practices through beekeeping management.

\section{INTRODUCCIÓN}

Las abejas melíferas (Apis mellifera L.) recolectan polen de las flores, lo mezclan con néctar y sus propias sustancias salivares y lo depositan en las corbículas de sus patas posteriores hasta formar masas de polen denominadas cargas corbiculares o cúmulos corbiculares (Montenegro et al., 1992; Sá-Otero et al., 2002; Baldi et al., 2004; Vit, 2009). Estas cargas son llevadas por las abejas a la colmena, donde las almacenan y maduran, para producir el pan de abejas, alimento esencial para el desarrollo y sostenimiento de sus colonias, especialmente para las crías (Keller et al., 2005). Los apicultores pueden colocar trampas caza-polen en las entradas de las colmenas, las cuales permiten el paso del cuerpo de la abeja pero impiden el paso de las cargas corbiculares, que caen en un cajón de recolección (García-García et al., 1998; Baldi et al., 2004; Vit, 2009). El polen recolectado mediante trampas caza-polen y posteriormente sometido a secado, limpieza y envasado se denomina polen apícola (Baldi et al., 2004; Vit, 2009).

Dentro de la flora utilizada por las abejas como fuente de polen se encuentran árboles, arbustos y palmas que habitan en bosques naturales y en otras áreas forestales (Marques-Souza, 1993; Sayas \& Huamán, 2009; Luz \& Barth, 2012); por tal razón, el polen apícola puede ser considerado como subproducto de los bosques y promoverse como producto forestal no maderable (PFNM). Según la FAO (2013) los PFNM son productos de origen biológico distintos de la madera, procedentes de los bosques, otras superficies boscosas y de árboles de fuera de los bosques. El reconocimiento del polen apícola como PFNM, permite considerar a la apicultura como una actividad que puede ser integrada al manejo y conservación de las especies forestales y de los ecosistemas donde éstas habitan (Porter-Bolland, 2003; Luz \& Barth, 2012). Si la apicultura tiene una directa relación con las coberturas boscosas, se puede convertir en una herramienta práctica que sensibilice a las comunidades sobre el valor de la biodiversidad, favoreciendo la inclusión de prácticas de conservación del bosque como parte del manejo apícola (Porter-Bolland, 2003; Bradbear, 2009). Además, los productos apícolas con origen botánico en los bosques pueden llegar fácilmente a los mercados, teniendo en cuenta las tendencias actuales hacia el consumo de productos naturales y amigables con el ambiente (Rodríguez, 2006; Bradbear, 2009). El polen apícola es el segundo producto de la colmena más consumido dadas sus propiedades nutricionales y medicinales, las cuales están vinculadas a su origen botánico (Vit, 2009).

En Colombia los bosques Andinos de la Cordillera Oriental se reconocen como uno de los ecosistemas prioritarios para adelantar acciones tendientes a su manejo y conservación, debido entre otros aspectos, a que constituyen el hábitat para el roble Quercus humboldtii Bonpl. (Fagaceae), especie en estado vulnerable y con veda nacional para su aprovechamiento forestal (Rodríguez et al., 2005; Salinas \& Cárdenas, 2007). Los PFNM se consideran un tema central para consolidar estas acciones, ya que las comunidades locales han incorporado el uso del bosque a sus sistemas de vida, ayudando a fortalecer su base económica y/o alimentaria (Ariza et al., 2010). De los productos apícolas, la miel ya se ha identificado como producto forestal no maderable de bosques de roble de la Cordillera Oriental (Cárdenas et al., 2000; Chamorro 2011) y existe un gran potencial para el polen apícola. La producción de polen apícola en la Cordillera Oriental es una de las más altas en el mundo (40 kg por colmena) y los bosques andinos son reconocidos por los apicultores de esta región como fuentes importantes de polen (Martínez, 2006). Sin embargo, para considerar al polen apícola como producto forestal no maderable se hace necesario conocer la contribución de la flora de los bosques andinos a la producción de polen apícola en la Cordillera Oriental, ya que las abejas en función de la floración disponible, seleccionan los recursos según su abundancia y accesibilidad, desplazándose a diferentes distancias, direcciones y coberturas, pero sin ninguna preferencia obvia por la vegetación más cercana a los apiarios (Montoya, 2011; Aronne et al., 2012). Por tal motivo el objetivo de este trabajo fue identificar 
el origen botánico de polen apícola proveniente de apiarios de la Cordillera Oriental que tienen bosques andinos en su área de influencia y determinar qué elementos de la flora de estas coberturas contribuyen de forma significativa en la producción de polen apícola.

\section{MATERIALES Y MÉTODOS}

\section{ÁREA DE ESTUDIO}

Se seleccionaron 13 apiarios con bosques andinos dentro de su área de influencia (menos de $1 \mathrm{~km}$ de radio), localizados en la vertiente occidental de la Cordillera Oriental de Colombia, en los departamentos de Cundinamarca, Boyacá y Santander, entre los 2500 y $3500 \mathrm{msnm}$ (tabla 1, figura 1). Los apiarios se encuentran ubicados en fincas donde predomina el potrero de kikuyo Penisetum clandestinum con hierbas exóticas como Hypochaeris radicata, Trifolium pratense y $T$. repens. También hay fragmentos de bosques andinos con roble $Q$. humboldtii y encenillo Weinmannia tomentosa, matorrales (Clethra sp., Dodonea viscosa, Morella spp.), cultivos (papa Solanum tuberosum, arveja Pisum sativum, haba Vicia fava, arvenses de la familia Brassicaceae) y plantaciones forestales (Eucalyptus globulus, Pinus spp.). Los bosques con roble en la mayoría de las localidades corresponden a fragmentos altamente intervenidos y en algunas zonas similares a una plantación forestal, sin estrato herbáceo o arbustivo y con evidencia de pastoreo. Los apicultores tienen sus apiarios en los matorrales que limitan con los fragmentos de bosques, con 10 colmenas en promedio dedicadas a la producción de miel, polen o a los dos productos. En general, según los apicultores, la producción de miel $(12 \mathrm{~kg}$ por colmena) se da en los primeros meses del año y la de polen (18 $\mathrm{kg}$ por colmena) durante la mayor parte del año, siendo en algunos apiarios mayor que la de miel.

Tabla 1. Origen botánico de las 25 muestras de polen apícola analizadas, provenientes de la Cordillera Oriental de Colombia. En la localidad se indica departamento, municipio y vereda. En la figura 1 se observa la ubicación geográfica de los apiarios estudiados

\begin{tabular}{|c|c|c|c|c|c|c|c|}
\hline \multicolumn{2}{|c|}{ Localidad } & Longitud & Latitud & $\operatorname{Altura(m)}$ & Cosecha & Clasificación & Tipos polínicos \\
\hline \multirow[b]{2}{*}{$\mathrm{U}$} & \multirow{2}{*}{$\begin{array}{l}\text { Cundinamarca, } \\
\text { Guachetá, } \\
\text { Santuario }\end{array}$} & \multirow[b]{2}{*}{$73^{\circ} 38^{\prime} 36.12^{\prime \prime}$} & \multirow[b]{2}{*}{$5^{\circ} 24^{\prime} 43.20^{\prime \prime}$} & \multirow{2}{*}{2985} & Ago 2008 & Monofloral & Trifolium pratense \\
\hline & & & & & Feb 2010 & Monofloral & Eucalyptus globulus \\
\hline \multirow{4}{*}{$\mathrm{S}$} & \multirow{4}{*}{$\begin{array}{l}\text { Cundinamarca, } \\
\text { Guachetá, } \\
\text { Peñas }\end{array}$} & \multirow{4}{*}{$73^{\circ} 38^{\prime} 29.58^{\prime \prime}$} & \multirow{4}{*}{$5^{\circ} 24^{\prime} 37.55^{\prime \prime}$} & \multirow{4}{*}{3075} & Mar 2008 & Polifloral & \\
\hline & & & & & Abr 2008 & Monofloral & H. radicata \\
\hline & & & & & Ago 2008 & Bifloral & $\begin{array}{l}\text { Tipo Brassicaceae, } H \text {. } \\
\text { radicata }\end{array}$ \\
\hline & & & & & Feb 2010 & Monofloral & Tipo Brassicaceae \\
\hline \multirow{3}{*}{$\mathrm{F}$} & \multirow{3}{*}{$\begin{array}{l}\text { Boyacá, } \\
\text { Ráquira, Firita } \\
\text { Peña Arriba }\end{array}$} & \multirow{3}{*}{$73^{\circ} 36^{\prime} 36.00^{\prime \prime}$} & \multirow{3}{*}{$5^{\circ} 25^{\prime} 22.80^{\prime \prime}$} & \multirow{3}{*}{3266} & May 2008 & Monofloral & H. radicata \\
\hline & & & & & Ago 2008 & Bifloral & $\begin{array}{l}\text { Tipo Brassicaceae, } H \text {. } \\
\text { radicata }\end{array}$ \\
\hline & & & & & Feb 2010 & Bifloral & $\begin{array}{l}\text { Tipo Brassicaceae, } \\
\text { Vallea stipularis }\end{array}$ \\
\hline A & $\begin{array}{l}\text { Boyacá, } \\
\text { Chiquinquirá, } \\
\text { Arboledas }\end{array}$ & $73^{\circ} 45^{\prime} 13.27^{\prime \prime}$ & $5^{\circ} 35^{\prime} 52.58^{\prime \prime}$ & 2677 & Dic 2009 & Bifloral & $\begin{array}{l}\text { E. globulus, Quercus } \\
\text { humboldtii }\end{array}$ \\
\hline $\mathrm{C}$ & $\begin{array}{l}\text { Boyacá, Paipa, } \\
\text { Marcura }\end{array}$ & $73^{\circ} 8^{\prime} 20.19^{\prime \prime}$ & $5^{\circ} 48^{\prime} 46.95^{\prime \prime}$ & 3132 & May 2009 & Monofloral & E. globulus \\
\hline $\mathrm{D}$ & $\begin{array}{l}\text { Boyacá, Paipa, } \\
\text { Medios }\end{array}$ & $73^{\circ} 6^{\prime} 57.01^{\prime \prime}$ & $5^{\circ} 49^{\prime} 29.00^{\prime \prime}$ & 2873 & May 2009 & Monofloral & E. globulus \\
\hline
\end{tabular}


Polen apícola como producto forestal no maderable

\begin{tabular}{|c|c|c|c|c|c|c|c|}
\hline \multicolumn{2}{|c|}{ Localidad } & \multirow{4}{*}{$\begin{array}{l}\text { Longitud } \\
72^{\circ} 51^{\prime} 46.90^{\prime \prime}\end{array}$} & \multirow{4}{*}{$\begin{array}{l}\text { Latitud } \\
5^{\circ} 58^{\prime} 44.56^{\prime \prime}\end{array}$} & \multirow{4}{*}{$\begin{array}{l}\text { Altura(m) } \\
3087\end{array}$} & \multirow{2}{*}{$\begin{array}{l}\text { Cosecha } \\
\text { May } 2008\end{array}$} & \multirow{2}{*}{$\begin{array}{l}\text { Clasificación } \\
\text { Monofloral }\end{array}$} & \multirow{2}{*}{$\begin{array}{l}\text { Tipos polínicos } \\
\text { Weinmannia tomentosa }\end{array}$} \\
\hline \multirow{3}{*}{$\mathrm{T}$} & \multirow{3}{*}{$\begin{array}{l}\text { Boyacá, Belen, } \\
\text { Tirinquita }\end{array}$} & & & & & & \\
\hline & & & & & May 2009 & Monofloral & E. globulus \\
\hline & & & & & Dic 2009 & Bifloral & $\begin{array}{l}\text { Tipo Asteraceae B (aff. } \\
\text { Baccharis), T. pratense }\end{array}$ \\
\hline & Boyacá, & \multirow[b]{2}{*}{$72^{\circ} 50^{\prime} 41.56^{\prime \prime}$} & \multirow[b]{2}{*}{ 6³'43.90”' } & \multirow[b]{2}{*}{3083} & May 2008 & Monofloral & Tipo Brassicaceae \\
\hline V & $\begin{array}{l}\text { Tutaza, } \\
\text { Cartavita }\end{array}$ & & & & May 2009 & Bifloral & $\begin{array}{l}\text { Tipo Brassicaceae, } H \text {. } \\
\text { radicata }\end{array}$ \\
\hline \multirow[t]{2}{*}{$\mathrm{H}$} & $\begin{array}{l}\text { Boyacá, } \\
\text { Susacón, El }\end{array}$ & \multirow[t]{2}{*}{$72^{\circ} 42^{\prime} 27.36^{\prime \prime}$} & \multirow[t]{2}{*}{$6^{\circ} 10^{\prime} 41.52^{\prime \prime}$} & \multirow[t]{2}{*}{2745} & Ago 2008 & Bifloral & $\begin{array}{l}\text { Q. humboldtii, } H \text {. } \\
\text { radicata }\end{array}$ \\
\hline & Hato & & & & May 2009 & Monofloral & Morella \\
\hline \multirow{2}{*}{ M } & Boyacá, Soatá, & \multirow{2}{*}{$72^{\circ} 43^{\prime} 30.72^{\prime \prime}$} & \multirow{2}{*}{$6^{\circ} 20^{\prime} 34.86^{\prime \prime}$} & \multirow{2}{*}{2601} & Ago 2008 & Monofloral & Tipo Brassicaceae \\
\hline & Molinos & & & & May 2009 & Monofloral & Q. humboldtii \\
\hline \multirow{2}{*}{$\mathrm{P}$} & Boyacá, & \multirow{2}{*}{$72^{\circ} 33^{\prime} 47.61^{\prime \prime}$} & \multirow{2}{*}{$6^{\circ} 21^{\prime} 39.74^{\prime \prime}$} & \multirow{2}{*}{2540} & Ene 2010 & Monofloral & Q. humboldtii \\
\hline & $\begin{array}{l}\text { Sañateo, } \\
\text { Peñuela }\end{array}$ & & & & Jul 2010 & Monofloral & H. radicata \\
\hline $\mathrm{E}$ & $\begin{array}{l}\text { Boyacá, San } \\
\text { Mateo, El } \\
\text { Palmar, }\end{array}$ & $72^{\circ} 33^{\prime} 28.80^{\prime \prime}$ & $6^{\circ} 25^{\prime} 40.80^{\prime \prime}$ & 2621 & Dic 2009 & Polifloral & \\
\hline $\mathrm{R}$ & $\begin{array}{l}\text { Santander, } \\
\text { Molagavita, } \\
\text { Potrero de } \\
\text { Rodríguez, }\end{array}$ & $72^{\circ} 46^{\prime} 32.01^{\prime \prime}$ & $6^{\circ} 39^{\prime} 50.17^{\prime \prime}$ & 2872 & Mar 2010 & Bifloral & Viburnum, H. radicata \\
\hline
\end{tabular}

\section{MUESTRAS DE POLEN APÍCOLA Y ANÁLISIS MELISOPALINOLÓGICO}

Se obtuvieron 25 muestras (250 g, cada una) de polen apícola, que fueron enviadas al Laboratorio de Melisopalinología del Departamento de Biología de Universidad Nacional de Colombia (Bogotá) por los apicultores después de la respectiva cosecha (recolección mediante trampas caza-polen y mezcla del polen producido por las colmenas de un apiario) y poscosecha (secado, limpieza y envasado). Las muestras provienen de cosechas de polen producidas entre marzo de 2008 y julio de 2010 (tabla 1). En el laboratorio se tomaron 5 g de cada muestra, los cuales se maceraron y homogenizaron en un mortero con alcohol al 70\%. De esta mezcla se tomó una submuestra de $0,1 \mathrm{~g}$, la cual se colocó en tubo de micro-centrífuga con ácido acético glacial (AAG) y se dejó en reposo por 24 horas. Después de transcurrido ese tiempo, se descartó el AAG y el sedimento polínico se sometió a acetólisis (Erdtman, 1960). Seguido se hicieron tres lavados, el primero en alcohol al $70 \%$, el segundo en agua destilada y el tercero en alcohol al 70\%. Al final se agregó glicerina y se tomaron dos alícuotas para realizar una preparación microscópica permanente (gelatina glicerinada y sellada con parafina) con dos áreas de conteo por lámina. Se utilizó un microscopio óptico para la identificación y conteo de 500 granos de polen por muestra, a 400 y 1000 aumentos. La identificación se llevó a nivel específico cuando fue posible. En caso contrario se llegó a nivel genérico o de tipo polínico que contiene géneros afines (Louveaux et al., 1978). Para algunos géneros $\mathrm{y}$ tipos polínicos se indican las especies y géneros a las que se puede atribuir el polen en cuestión (Ortíz, 1989). El reconocimiento taxonómico se hizo con ayuda de catálogos de polen (Roubik \& Moreno, 1991; Velásquez, 1999; Bogotá, 2002), así como por comparación directa con la palinoteca del Laboratorio de Investigaciones en Abejas de la Universidad Nacional de Colombia (LABUN). En esta palinoteca hay polen de referencia de plantas 
visitadas por abejas encontradas alrededor de los apiarios estudiados en este trabajo.

Para definir el origen botánico de cada muestra, se expresó la abundancia de cada tipo polínico en valor relativo (\%) y se clasificó en polen dominante " $D$ " cuando la abundancia relativa fue mayor a $45 \%$, polen secundario "S" cuando se encontró entre 16 y $45 \%$, polen aislado importante "AI" cuando abundó entre 3 y $15 \%$, polen aislado "A" cuando se encontró entre 1 y $3 \%$ y polen esporádico "E" cuando se encontró en menos de 1\% (Louveaux et al., 1978 modificadas en este trabajo). Con base en lo anterior la muestra se tipificó como monofloral (un tipo polínico dominante), bifloral (dos tipos polínicos secundarios) o polifloral (un sólo tipo polínico secundario y los demás aislados o todos los tipos polínicos aislados). También se calculó la frecuencia de aparición "FA" de cada tipo polínico en las 25 muestras analizadas.

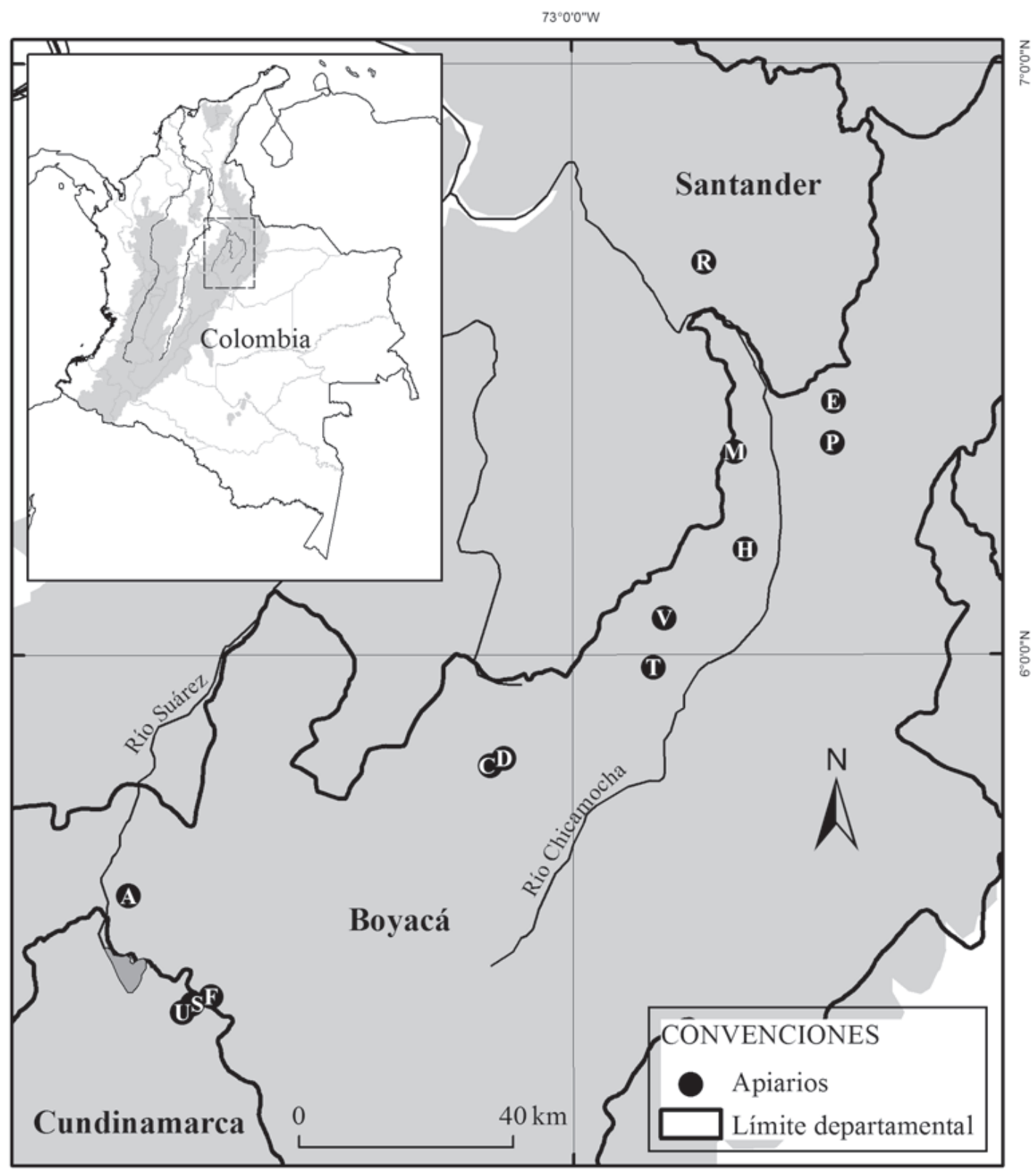

Figura 1. Ubicación geográfica de los apiarios estudiados. En la tabla 1 se observa en forma detallada cada una de las localidades. 


\section{RESULTADOS}

Se identificaron 63 tipos polínicos pertenecientes a 41 familias botánicas (tabla 2), de las cuales Asteraceae y Fabaceae fueron las mejores representadas con nueve y seis tipos polínicos, respectivamente. Los principales tipos polínicos encontrados se muestran en la figura 2. De los 63 tipos polínicos identificados, se destacaron por su alta frecuencia de aparición y por clasificarse como dominantes y secundarios $H$. radicata, tipo Brassicaceae que incluye a Brassica y Raphanus, T. pratense, E. globulus y $Q$. humboldtii (tabla 2). Otros tipos polínicos poco frecuentes pero importantes porque se encontraron dominantes o secundarios fueron: Viburnum, Morella, W. tomentosa, Vallea stipularis y Asteraceae B (aff. Baccharis) (tablas 1 y 2). Igualmente, dentro de los recursos acompañantes de las fuentes dominantes y secundarias se destacaron por su frecuencia de aparición Acacia decurrens, Asteraceae C (aff. Chromolaena), Hypericum, Gaiadendron punctatum, Muehlenbeckia tamnifolia, tipo Rosaceae que incluye Rubus y Hesperomeles, Asteraceae S (aff. Senecio) y $T$. repens (tablas 1 y 2 ).

De las 25 muestras analizadas, 15 muestras se clasificaron como monoflorales, ocho como biflorales y dos como poliflorales, con predominio de cuatro fuentes de polen: E. globulus (cuatro muestras monoflorales y una bifloral), $H$. radicata (tres muestras monoflorales y cinco biflorales), tipo Brassicaceae (tres muestras monoflorales y cuatro biflorales) y $Q$. humboldtii (dos muestras monoflorales y dos biflorales; tabla 1).

Tabla 2. Tipos polínicos identificados en las muestras de polen apícola. Se indica la frecuencia de aparición (FA) y el número de muestras en la que cada tipo polínico se clasificó como $\mathrm{D}=$ Dominante $(>45 \%)$, $\mathrm{S}=$ secundario $(16-45 \%)$, IA = importante aislado (3-15\%), A= aislado (1-3\%) y E= esporádico $(<1 \%)$ * Nativa, ** Introducida

\begin{tabular}{|c|c|c|c|c|c|c|c|}
\hline Familia & Tipo polínico & D & $\mathbf{S}$ & IA & $\mathbf{A}$ & $\mathbf{E}$ & FA $\%$ \\
\hline Adoxaceae & Viburnum (V.tinoides, V. triphyllum)* & - & 2 & 5 & 2 & 6 & 60 \\
\hline Amaranthaceae & Tipo Amaranthaceae (Amaranthus) & - & - & - & - & 1 & 4 \\
\hline Apiaceae & Apiaceae & - & - & 1 & - & - & 4 \\
\hline Aquifoliaceae & Ilex* & - & - & - & 1 & 4 & 20 \\
\hline Araliaceae & Oreopanax* & - & - & - & - & 2 & 8 \\
\hline \multirow{10}{*}{ Asteraceae } & Araliaceae & - & - & - & - & 1 & 4 \\
\hline & Hypochaeris radicata** & 3 & 8 & 11 & 2 & 1 & 100 \\
\hline & Asteraceae S (aff. Senecio) & - & - & 3 & 3 & 3 & 36 \\
\hline & Taraxacum officinale ${ }^{* *}$ & - & - & - & 1 & 6 & 28 \\
\hline & Asteraceae B (aff Baccharis) & - & 1 & 1 & 5 & 1 & 32 \\
\hline & Eirmocephala & - & - & - & - & 7 & 28 \\
\hline & Asteraceae P (aff. Pentacalia) & - & - & - & 1 & 4 & 20 \\
\hline & Asteraceae C (aff Chromolaena) & - & - & 3 & 2 & - & 20 \\
\hline & Asteraceae V (aff. Verbesina) & - & - & - & 2 & 2 & 16 \\
\hline & Vernonia & - & - & 1 & 2 & - & 12 \\
\hline Balsaminaceae & Impatiens & - & - & - & - & 1 & 4 \\
\hline Betulaceae & Alnus acuminata* & - & - & - & 1 & - & 4 \\
\hline Boraginaceae & Cordia* & - & - & - & - & 4 & 16 \\
\hline Brassicaceae & Tipo Brassicaceae (Brassica, Raphanus)** & 3 & 5 & 4 & 4 & 2 & 72 \\
\hline Bromeliaceae & Puya* & - & - & 1 & - & 7 & 32 \\
\hline Chloranthaceae & Hedyosmun* & - & - & - & 1 & - & 4 \\
\hline Clusiaceae & Clusia* & - & - & 1 & - & - & 4 \\
\hline
\end{tabular}


Fermín J. Chamorro-García, Daniela León-Bonilla \& Guiomar Nates-Parra

\begin{tabular}{|c|c|c|c|c|c|c|c|}
\hline Familia & Tipo polínico & D & $\mathbf{S}$ & IA & A & $\mathbf{E}$ & FA\% \\
\hline Cunoniaceae & Weinmannia tomentosa* & 1 & - & 1 & - & 2 & 16 \\
\hline Elaeocarpaceae & Vallea stipularis* & - & 1 & - & - & - & 4 \\
\hline Ericaceae & Ericaceae* & - & - & - & - & 3 & 12 \\
\hline Escalloniaceae & Escallonia* & - & - & - & 2 & 1 & 12 \\
\hline \multirow{7}{*}{$\begin{array}{l}\text { Euphorbiaceae } \\
\text { Fabaceae }\end{array}$} & Ricinus comunis** & - & - & - & - & 1 & 4 \\
\hline & Trifolium repens** & - & - & 10 & 4 & 8 & 88 \\
\hline & Trifolium pratense ${ }^{* *}$ & 1 & 2 & 13 & 3 & 2 & 84 \\
\hline & Acacia decurrens** & - & - & 3 & 2 & 3 & 32 \\
\hline & Ulex europaeus** & - & - & - & 3 & 3 & 24 \\
\hline & Desmodium* & - & - & - & - & 2 & 8 \\
\hline & Erythrina* & - & - & - & - & 1 & 4 \\
\hline Fagaceae & Quercus humboldtii* & 2 & 3 & 3 & 2 & 6 & 64 \\
\hline Hypericaceae & Hypericum* & - & - & 3 & 1 & 1 & 20 \\
\hline Lamiaceae & Lamiaceae & - & - & - & - & 1 & 4 \\
\hline Lauraceae & Lauraceae & - & - & - & - & 1 & 4 \\
\hline Loranthaceae & Gaiadendron punctatum* & - & - & 3 & 4 & 2 & 36 \\
\hline Malvaceae & Sida* & - & - & - & - & 1 & 4 \\
\hline Melastomataceae & Melastomataceae & - & - & 1 & - & 2 & 12 \\
\hline Myricaceae & Morella (M. parvifolia, M. pubescens)* & 1 & - & 4 & 2 & 2 & 36 \\
\hline \multirow[t]{2}{*}{ Myrtaceae } & Eucalyptus globulus** & 4 & 2 & 10 & 3 & 1 & 80 \\
\hline & Tipo Myrtaceae (Myrcia, Myrcianthes)* & - & - & 1 & - & - & 4 \\
\hline Oleaceae & Fraxinus chinensis ${ }^{* *}$ & - & - & - & 1 & 6 & 28 \\
\hline Onagraceae & Ludwigia* & - & - & - & - & 2 & 8 \\
\hline \multirow[t]{2}{*}{ Passifloraceae } & Passiflora* & - & - & - & 1 & 4 & 20 \\
\hline & Passiflora bogotensis* & - & - & - & - & 1 & 4 \\
\hline Pinaceae & Pinus $^{* *}$ & - & - & - & - & 1 & 4 \\
\hline Piperaceae & Piper* & - & - & 1 & - & - & 4 \\
\hline \multirow[t]{2}{*}{ Poaceae } & Holcus lanatus** & - & - & - & 1 & 2 & 12 \\
\hline & Poaceae & - & - & - & - & 1 & 4 \\
\hline \multirow[t]{2}{*}{ Polygonaceae } & Muehlenbeckia tamnifolia* & - & - & 3 & 4 & 3 & 40 \\
\hline & Polygonum* & - & - & - & - & 3 & 12 \\
\hline Rosaceae & Tipo Rosaceae (Rubus, Hesperomeles)* & - & - & 4 & 7 & 3 & 56 \\
\hline \multirow{4}{*}{ Rubiaceae } & Prunus & - & - & 1 & - & - & 4 \\
\hline & Spermacoce* & - & - & - & - & 5 & 20 \\
\hline & Arcytophyllum* & - & - & - & - & 3 & 12 \\
\hline & Psychotria* & - & - & - & - & 1 & 4 \\
\hline Salicaceae & Salix & - & - & 1 & - & - & 4 \\
\hline \multirow[t]{2}{*}{ Solanaceae } & Solanaceae (Solanum) & - & - & - & 1 & 2 & 12 \\
\hline & Brugmansia* & - & - & - & - & 1 & 4 \\
\hline Symplocaceae & Symplocos* & - & - & - & - & 2 & 8 \\
\hline Indeterminado & Indeterminado & - & - & - & - & 1 & 4 \\
\hline
\end{tabular}




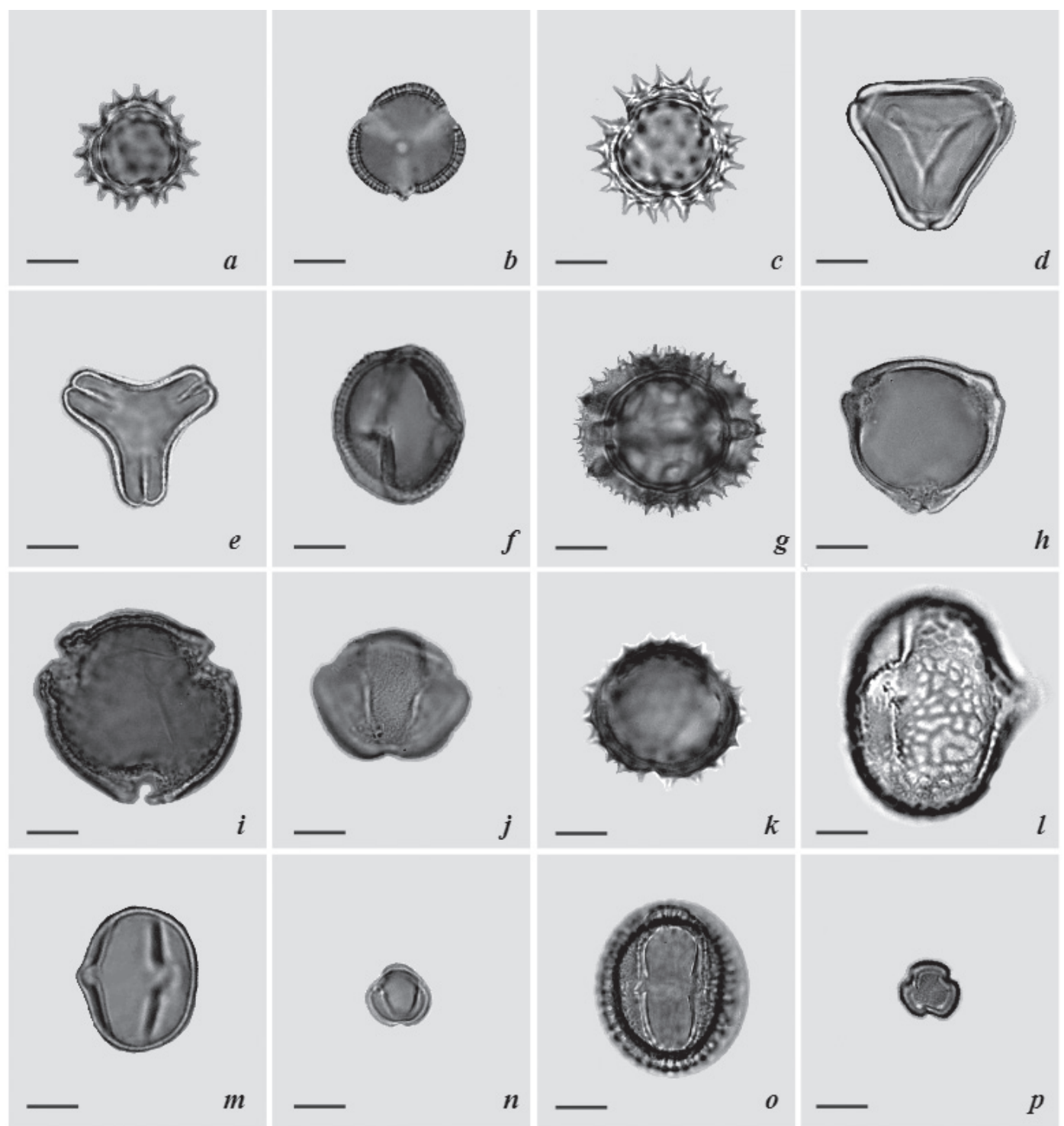

Figura 2. Fotomicrografías de los tipos polínicos más abundantes y frecuentes en las muestras de polen apícola. a. Asteraceae B (aff. Baccharis), b. Tipo Brassicaceae, c. Asteraceae C (aff. Chromolaena), d. Eucalyptus globulus, e. Gaiadendron punctatum, f. Hypericum strictum, g. Hypochaeris radicata, h. Morella, i. Quercus humboldtii, j. Tipo Rosaceae, $\boldsymbol{k}$. Asteraceae S (aff. Senecio), l. Trifolium pratense, $\boldsymbol{m}$. Trifolium repens, $\boldsymbol{n}$. Vallea stipularis, o. Viburnum, $\boldsymbol{p}$. Weinmannia tomentosa. Barra de escala $=10 \mu \mathrm{m}$.

\section{DISCUSIÓN}

El espectro polínico del polen apícola analizado muestra en general una baja contribución de la flora de los bosques andinos y una alta dominancia de plantas exóticas (principalmente $H$. radicata, tipo Brassicaceae y E. globulus) en la producción apícola. No obstante, elementos florísticos nativos de gran importancia ecológica en los bosques andinos, por su dominancia, frecuencia y densidad, como Q. humboldtii, Morella (M. parvifolia, M. pubescens), V. stipularis, Viburnum (V. tinoides, $V$. triphyllum) y $W$. tomentosa (Marin-Córdoba \& Betancur, 1997; Devia \& Arenas, 2000; Cortés, 2003; Pulido et al., 2006; Montes, 2011), sobresalieron por su abundancia relativa dominante y secundaria en los muestras de polen apícola (Tabla 2). En particular se destacó Q. humboldtii con una frecuencia de aparición superior al $50 \%$ y clasificado como dominante en dos muestras, 
secundario en tres muestras e importante aislado en tres muestras (Tabla 2). El género Quercus se encuentra entre las fuentes de polen más utilizadas por las abejas melíferas (Ortíz, 1990; Keller et $a l ., 2005)$ y resultados similares a los encontrados para $Q$. humboldtii, en términos de frecuencia y abundancia, ya se han registrado para otras especies de roble ( $Q$. rotundifolia, $Q$. suber, $Q$. coccifera, Q. canariensis y Q. faginea) (Ortiz et al., 1990; Ortiz 1994; Hidalgo et al., 1996; Díaz et al., 1997). Incluso desde una perspectiva productiva y comercial, las especies del género Quercus se han registrado como una de las fuentes de polen con mayor contribución dentro del polen apícola en España (Arroyo et al. 1986; Sá-Otero et al., 2002). Las abejas recolectan el polen de roble (Quercus spp.) de forma frecuente y en abundancia, debido a la gran cantidad de flores y polen que producen estas plantas y que facilitan la recolección del recurso de forma eficiente (Hidalgo et al., 1996; Tormo et al., 1996; Aronne et al., 2012).

Con respecto a Morella, V. stipularis, Viburnum y $W$. tomentosa, se mencionan como fuentes de polen apícola en algunas zonas de Boyacá (Salamanca-Grosso et al., 2011) y especies como $W$. tomentosa también se ha reconocido como planta de importancia melífera (Cárdenas et al., 2000; Mahecha et al., 2004) y asociada a cosechas de mieles monoflorales (Nates-Parra et al., 2011). Adicionalmente, es importante mencionar que las ramas de $M$. parvifolia y $M$. pubescens son utilizadas para elaboración de cruces durante la celebración religiosa de la Cruz de Mayo, actividad que produce un grave impacto en las poblaciones de estas especies (Parra, 2003). Como estrategia para desincentivar o disminuir la extracción de ramas de plantas silvestres de Morella, se puede promover el uso apícola de estas especies, así las comunidades pueden aprovecharlas para la producción de polen y mantener su uso tradicional.

Por otra parte, hay que destacar el aporte de polen del tipo Rosaceae (Rubus, Hesperomeles) y de la familia Asteraceae, fuentes de recursos que pueden estar presentes en los bosques andinos o en los bordes de estos (Mahecha et al., 2004; Pulido et al., 2006; Cancino-Escalante et al., 2011). Las plantas de las familias Rosaceae y Asteraceae presentan flores de fácil acceso para las abejas, de las cuales estos insectos pueden obtener no sólo polen sino también néctar (Chamorro, obs. pers.). En la Sabana de Bogotá, Rubus también se ha registrado dentro de las plantas nativas como fuente polinífera (Montoya, 2011) y en España y Chile, Rubus ulmifolius es preferida por las abejas como fuente de polen en determinados momentos del año (Montenegro et al., 1992; Díaz et al., 1995). Incluso en España se producen mieles monoflorales de Rubus (Escuredo et al., 2011). En cuanto a Asteraceae, su importancia como fuente de polen ha sido registrada en diferentes regiones (Argentina: Faye et al., 2002; México: Villanueva, 2002; Colombia: Vivas et al., 2008, Montoya, 2011; Brasil: Modro et al., 2011) y aunque no fue posible identificar a nivel específico la mayoría de sus tipos polínicos, es muy probable que estos correspondan a especies presentes en áreas en regeneración. Por ejemplo, Baccharis latifolia, distribuida ampliamente en la Cordillera Oriental, es típica de bosques en regeneración (Castañeda et al., 2007) y en áreas andinas de Perú y Colombia se ha registrado como fuente importante de polen (Sayas \& Huamán, 2009; Salamanca-Grosso et al., 2011).

Con el registro de $Q$. humboldtii y otros especies nativas (Morella spp., V. stipularis, Viburnum spp. y $W$. tomentosa) como fuente de polen para la producción de polen apícola en la Cordillera Oriental, se agrega valor al uso apícola como alternativa no maderable para los bosques andinos y específicamente para $Q$. humboldtii, que también se ha identificado como fuente primaria de miel de mielato en algunas zonas de Boyacá (Chamorro, 2011). Además complementa los usos no maderables (principalmente medicinal, tintes y colorantes) registrados por Ariza et al. (2010) para estas especies. Sin embargo, es necesario realizar un seguimiento fenológico de la floración de estas especies y su uso como fuente de polen por las abejas, con el fin de definir con claridad los periodos de producción de polen de estas especies e implementar medidas de manejo apícola que permitan asegurar la producción y diferenciación de estos productos. 


\section{CONCLUSIONES}

Existe un alto potencial para la promoción del polen apícola como producto no maderable de los bosques andinos en la Cordillera Oriental, gracias a que las abejas recolectan polen de forma abundante de algunas plantas comunes y características de estos ecosistemas ( $Q$. humboldtii, Morella spp., $V$. stipularis, Viburnum spp. y $W$. tomentosa). Específicamente, $Q$. humboldtii puede constituirse en un recurso de importancia polinífera y con potencial para la cosecha de polen apícola monofloral. En este contexto, la apicultura se convierte entonces en una alternativa viable de uso no maderable de los bosques andinos, al encontrarse una relación directa entre ecosistemas y la producción apícola, en términos de la identificación de elementos florísticos apícola que tienen como hábitat los bosques andinos y que contribuyan de forma significativa con la producción polen apícola. De esta forma, la producción de polen apícola con un origen particular en los bosques andinos puede ser una alternativa económica para desincentivar la extracción de maderables, en especial del roble y al mismo tiempo puede generar desarrollo rural a través de la incursión de las comunidades locales en los mercados diferenciados. Con los resultados de este trabajo, se espera proporcionar a los actores relacionados con el sector forestal con la motivación suficiente para evaluar a la apicultura como una actividad comercial de protección viable, que puede ser integrada a los programas de conservación de bosques andinos.

\section{AGRADECIMIENTOS}

Esta investigación hace parte de los resultados del trabajo de grado de Fermín J. Chamorro, como parte de los requisitos para titularse como Especialista en Gerencia de Recursos Naturales de la Universidad Distrital Francisco José de Caldas. Este trabajo fue posible gracias al apoyo financiero de la Universidad Nacional de Colombia, Sede Bogotá y el Ministerio de Agricultura y Desarrollo Rural, a través del proyecto de investigación Diferenciación por origen botánico de mieles y polen de cuatro especies de abejas en Colombia concedido a Guiomar Nates Parra (Contrato No.2007C3476 118-584/2007). Agradecemos los valiosos comentarios de los evaluadores anónimos que contribuyeron a aclarar y mejorar la calidad de este trabajo.

\section{REFERENCIAS BIBLIOGRÁFICAS}

Ariza, W., Huertas, C., Hernández, A., Geltvez, J., González, J., \& López, L. (2010). Caracterización y usos tradicionales de productos forestales no maderables (PFNM) en el Corredor de Conservación Guantiva - La Rusia - Iguaque. Colombia Forestal, 13(1), 117-140.

Aronne, G., Giovanetti, M., Guarracino, M.R., \& De Micco, V. (2012). Foraging rules of flower selection applied by colonies of Apis mellifera: ranking and associations of floral sources. Functional Ecology, 26(5), 1186-1196.

Arroyo, J., Devesa, J.A., Herrera, F.J., Ortiz, P.L. \& Talavera, S. (1986). Flora Melitófila en Andalucía Occidental. Vida Apícola 18: 33-39.

Baldi, B., Grasso, D., Chaves, S., \& Fernandez, G. (2004). Caracterización bromatológica del polen apícola argentino. Ciencia, Docencia y Tecnología, 15(29), 145-181.

Bogotá, R.G. (2002). El polen de la subclase Asteridae en el páramo de Monserrate. Bogotá: Universidad Distrital Francisco José de Caldas, Centro de Investigaciones y Desarrollo Científico. 128 p.

Bradbear, N. (2009). Bees and their roles in forest livelihoods: A guide to the services provided by bees and the sustainable harvesting, processing and marketing of their products. Series Non-Wood Forest Products 19. Rome: Food and Agriculture Organization of the United Nations-FAO. $180 \mathrm{p}$. 
Cancino-Escalante, G.O., Sánchez-Montaño, L.R., Quevedo-García, E., \& DíazCarvajal, C. (2011). Caracterización fenotípica de accesiones de especies de Rubus L. de los municipios de Pamplona y Chitagá, Región Nororiental de Colombia. Universitas Scientiarum, 16 (3), 219-233.

Cárdenas, F., Devia, C.A. Cordero, H., Farah, M., Rojas, A., Herrera, J. C., \& Mesa, G. (2000). La Conservación y la producción por parte de las comunidades locales en la cuenca media del Río Chicamocha (Boyacá, Colombia). Bogotá, D.C.: Pontificia Universidad Javeriana, Instituto de Estudios Ambientales para el Desarrollo-IDEADE. 101 p.

Castañeda, S.L., Garzón, A.E., Cantillo, M.A., Torres, M.P., \& Silva, L.J. (2007). Análisis de la respuesta de ocho especies nativas del bosque alto andino ante dos métodos de propagación. Colombia Forestal, 10 (2), 79-90.

Chamorro, F.J. (2011). Caracterización de la miel y el polen como productos forestales no maderables de los bosques de roble en Boyacá, Colombia (Trabajo de grado, Especialización en Gerencia de Recursos Naturales). Bogotá, D.C.: Universidad Distrital Francisco José de Caldas, Facultad del Medio Ambiente y Recursos Naturales. $71 \mathrm{p}$.

Cortés, S. (2003). Estructura de la vegetación arbórea y arbustiva en el costado oriental de la Serranía de Chía (Cundinamarca, Colombia). Caldasia, 25 (1), 119-137.

Devia, C., \& Arenas, H. (2000). Evaluación del estatus ecosistémico y de manejo de los bosques de fagáceas (Quercus humboldtii y Trigonobalanus excelsa) en el norte de la Cordillera Oriental (Cundinamarca, Santander y Boyacá). En Cárdenas, F. (ed). Desarrollo Sostenible en los Andes de Colombia (Provincias de Norte, Gutiérrez y Valderrama) Boyacá, Colombia (pp. 6377). Bogotá: Pontificia Universidad Javeriana, Instituto de Estudios Ambientales para el Desarrollo-IDEADE.

Díaz, E., González, A., Fernández, E., \& Saá, M.P. (1995). Contribución al estudio de la utilización selectiva por Apis mellifera L. de la flora local en un colmenar del NW de la Península Ibérica (Galicia). Acta Botánica Malacitana, 20, 115-122.

Díaz, E., González, A., \& Saá, M.P. (1997). Estudio melisopalinológico en Galicia (NW de España). Orsis: Organismes i Sistemes, 12, 27-38.

Erdtman, G. (1960). The acetolysis method: a revised description. Sv Bot Tidskr Lund, 54 (4), 561-564.

Escuredo, O., Seijo, M.C., \& González, M.F. (2011). Descriptive analysis of Rubus honey from the north-west of Spain. International Journal of Food Science and Technology, 46, 2329-2336.

FAO (2013). Productos forestales no madereros. Organización de las Naciones Unidas para la Agricultura y la Alimentación-FAO. Disponible en: www.fao.org/forestry/ site/6388/es (Fecha de revisión: 4 de mayo de 2013).

Faye, P., Planchuelo, A., \& Molinelli, M. (2002). Relevamiento de la flora apícola e identificación de cargas de polen en el sureste de la provincia de Córdoba, Argentina. Agriscientia, 19, 19-30.

García-García, M. C., P.L., Ortiz \& M.J. Díez. (1998). Recolección de polen durante un día por Apis mellifera L. (Hymenoptera, Apidae), II. Lagascalia 20 (2): 195-210.

Hidalgo, M. I., Bootello, M.L., \& Pacheco, J. (1990). Origen floral de las cargas de polen recogidas por Apis mellifera L. en Alora

Colombia Forestal Vol. 16(1) / enero - junio, 2013 
(Málaga). Acta Botánica Malacitana, 15, 33-44.

Hidalgo, M. I., Recio, M., \& Cabezudo, B. (1996). Producción de polen en un matorral del Sur de España. Acta Botánica Malacitana, 21, 49-55.

Keller, I., Fluri, P., \& Imdorf, A. (2005). Pollen nutrition and colony development in honey bees: part I. Bee World, 86 (1), 3-10.

Louveaux, J., Maurizio, A., \& Vorwohl, G. (1978). Methods of melissopalynology. Bee World, 59, 139-157.

Luz, C.F.P., \& Barth, O.M. (2012). Pollen analysis of honey and beebread derived from Brazilian mangroves. Brazilian Journal of Botany, 35(1), 79-85.

Mahecha, G., Ovalle, A., Camelo, D., Rozo, A., \& Barrero, D. (2004). Vegetación del territorio CAR: 450 especies de sus llanuras y montañas. Bogotá, D.C.: Corporación Autónoma Regional de CundinamarcaCAR. $871 \mathrm{p}$.

Marín-Córdoba, C., \& Betancur, J. (1997). Estudio florístico en un robledal del Santuario de flora y fauna de Iguaque (Boyacá, Colombia). Revista de la Academia Colombiana de Ciencias Exactas, Físicas y Naturales, 21(8), 249-259.

Marques-Souza, A.C., Absy, M.L., Condé, P.A.A., \& Coelho, H.A.. (1993). Dados da obtenção de pólen por operárias de Apis mellifera no município de Ji-Paraná (RO), Brasil. Acta Amazônica, 23, 59-76.

Martínez, T. (2006). Diagnóstico de la actividad apícola y de la crianza de abejas en Colombia. Bogotá, D.C.: Ministerio de Agricultura y Desarrollo Rural-MADR, Instituto Interamericano de Cooperación para la Agricultura-IICA. 115 p.
Modro A., Marchini, L.C., \& Carmello, A. (2011). Origem botánica de cargas de pólen de colmeias de abelhas africanizadas em Piracicaba, SP. Ciência Rural, 41 (11), 1944-1951.

Montenegro, G., Gómez, M., \& Ávila, G. (1992). Importancia relativa de especies cuyo polen es utilizado por Apis mellifera en el área de la reserva nacional Los Ruiles, VII región de Chile. Acta Botánica Malacitana, $17,167-174$.

Montes, C.R. (2011). Estado del conocimiento en Weinmannia tomentosa L.f. (encenillo) y algunas propuestas de estudio sobre su regeneración. Revista de Investigación Agraria y Ambiental, 2 (1), 45-53.

Montoya, P.M. (2011). Uso de recursos florales poliníferos por Apis mellifera (Hymenoptera: Apidae) en apiarios de la Sabana de Bogotá y alrededores (Tesis, Maestría en Ciencias-Biología). Bogotá, D.C.: Universidad Nacional de Colombia, Sede Bogotá, Facultad de Ciencias. 66 p.

Nates-Parra, G., Montoya, P.M., Obregón, D., Chamorro, F.J., Ramírez, N., Giraldo, M.C. \& Solarte, V. (2011). Diferenciación por origen botánico de mieles y polen de cuatro especies de abejas en Colombia (Informe Final Proyecto de Investigación). Bogotá, D.C.: Laboratorio de Investigaciones en Abejas-LABUN, Facultad de Ciencias, Universidad Nacional de Colombia, Sede Bogotá. 214 p.

Ortiz, P.L. (1989). Melitopalinología en Andalucía Occidental (Tesis Doctoral). Sevilla, España: Universidad de Sevilla.

Ortiz, P.L., Fernández, I., \& Martín, M. (1990). Estudio Melitopalinológico en la Comarca de Aracena (Huelva). Lagascalia, 16 (1), 61-76. 
Ortiz, P.L. (1990). Aportación melitopalinológica al conocimiento de la flora apícola del Norte de Córdoba. Lagascalia, 15 (2), 165 170.

Ortiz, P.L. (1994). El polen recogido por Apis mellifera L. en Hinojos (Huelva) durante la primavera. Acta Botánica Malacitana, $19,115-122$.

Parra, C. (2003). Revisión taxonómica de la familia Myricaceae en Colombia. Caldasia, 25(1), 23-64.

Porter-Bolland, L. (2003). La apicultura y el paisaje maya. Estudio sobre la fenología de floración de las especies melíferas y su relación con el ciclo apícola en La Montaña, Campeche, México. Estudios Mexicanos, 19 (2), 303-330.

Pulido, M.T., Cavelier, J., \& Cortés, S.P. (2006). Structure and Composition of Colombian Montane Oak Forests. En Kapelle, M. (ed). Ecology and Conservation of Neotropical Montane oak forests. Ecological Studies 185 (pp. 141-151). Berlin: Springer.

Rodríguez, N., Rincón, A., Armenteras, D., Mendoza, H., Umaña, A.M., Arango, N., \& Baptiste, M.P. (2005). Corredor nororiental de robles: indicadores de estado de la biodiversidad, factores antrópicos asociados y áreas prioritarias de conservación. Serie: Indicadores de Seguimiento y Evaluación de la Política de Biodiversidad. Bogotá, D.C.: Instituto de Investigación de Recursos Biológicos Alexander von Humboldt. 88 p.

Rodríguez, M., (2006). Oportunidades comerciales para productos apícolas. Bogotá, D.C.: Instituto de Investigación de Recursos Biológicos Alexander von Humboldt. 85 p.

Roubik, D.W., \& Moreno, J.E. (1991). Pollen and Spores of Barro Colorado Island. Monographs in Systematic Botany 36. St.
Louis (USA): Missouri Botanical Garden. $268 \mathrm{p}$.

Sá-Otero, M. P., Bugarín, S.M., ArmestoBaztán, S., \& Díaz Losada, E. (2002). Método de determinación del origen geográfico del polen apícola comercial. Lazaroa, 23, 25 -34.

Salamanca Grosso, G., Osorio, M.P., \& Gutiérrez, A.M. (2011). Sistema trazable en el proceso de extracción y beneficio del polen corbicular colectado por Apis mellifera L. (Hymenoptera:Apidae) en la zona Altoandina de Boyacá, Colombia. Zootecnia Tropical, 29 (1), 127-138.

Salinas, N., \& Cárdenas, D. (2007). Roble: Quercus humboldtti. En Cárdenas, D. \& Salinas, N. (Eds.). Libro rojo de plantas de Colombia. Volumen 4. Especies maderables amenazadas: Primera parte (p. 23-29). Bogotá: Instituto Amazónico de Investigaciones Científicas (SINCHI), Ministerio de Ambiente, Vivienda y Desarrollo Territorial.

Sayas, R., \& Huamán, L. (2009). Determinación de la Flora Polinífera del valle de Oxapampa (Pasco-Perú) en base a estudios palinológicos. Ecología Aplicada, 8 (2), 53-59.

Tormo, R., Muñoz, A., Silva, I., \& Gallardo, F. (1996). Pollen production in anemophilous trees. Grana, 35, 38-46.

Velásquez, C. (1999). Atlas palinológico de la flora vascular paramuna de Colombia: Angiospermae. Medellín: Universidad Nacional de Colombia, Sede Medellín. $173 \mathrm{p}$.

Villanueva-G., R. (2002). Polliniferous plants and foraging strategies of Apis mellifera (Hymenoptera: Apidae) in the Yucatán Peninsula, Mexico. Revista de Biología Tropical, 50 (3/4), 1035-1043. 
Vit, P. (2009). Origen botánico y propiedades medicinales del polen apícola. Revista Médica de la Extensión Portuguesa ULA, 3 (1), 27-34.
Vivas, N., Maca, J., \& Pardo, M. (2008). Caracterización cualitativa del polen recolectado por Apis mellifera L. en tres apiarios del municipio de Popayán. Facultad de Ciencias Agropecuarias, 6 (2), 94-98. 